\title{
DESIGN AND ANALYSIS OF A MULTIVARIABLE FRACTIONAL ORDER CONTROLLER FOR A NON-MINIMUM PHASE SYSTEM
}

\author{
Cristina I. Muresan*, Eva H. Dulf*, Cosmin Copot ${ }^{* *}$, Robin De Keyser**, Clara Ionescu* \\ ${ }^{*}$ Technical University of Cluj-Napoca, Romania \\ ${ }^{* *}$ University of Ghent, Ghent, Belgium
}

\begin{abstract}
Two control strategies for multivariable processes are proposed that are based on a decentralised and a steady state decoupling approach. The designed controllers are fractional order PIs. The efficiency and robustness of the proposed strategies is tested and validated using a non-minimum phase process. Previous research for the same non-minimum phase process has proven that simple decentralised or decoupling techniques do not yield satisfactorily results and a multivariable IMC controller has been proposed as an alternative solution. The simulation results presented in this paper show that the proposed fractional order multivariable control strategies ensure an improved closed loop performance and disturbance rejection, as well as increased robustness to modelling uncertainties, as compared to traditional multivariable IMC controllers.
\end{abstract}

KEYWORDS: fractional order control, non-minimum phase system, water tanks, decentralized control, decoupling control, Internal Model Control

\section{INTRODUCTION}

The large majority of chemical processes are multivariable in nature, exhibiting some strong couplings and occasionally a non-minimum phase character that makes the control design problem a challenging task [1,2]. In general, for such systems, the objective of a control system is to maintain several controlled variables at independent set points. Despite the coupling problems associated with multivariable systems, a non-minimum phase system is even more difficult to control. None of the techniques that are based upon model inversion can be used since such an inversion leads to an unstable closed loop system. Multivariable controllers have been previously designed for such systems. However, simplified algorithms are generally preferred. In contrast to the centralised multivariable control, decentralised control is widely preferred in practice and industrial applications especially because of its main advantage that allows for an easy implementation and tuning, if a sufficient number of sensors and actuators exist. It is also highly reliable.

For highly interacting processes, a decoupling control is usually preferred instead of a decentralized algorithm. Decoupling is a procedure that reduces multivariable interactions [3] and sets the premises for an improved design of the decentralized control. The mathematical procedure to decouple a MIMO system consists in a transformation of the original transfer function matrix of the process into a diagonal one, achieved by using an additional controller, also called a decoupler, which is designed in order to compensate for process interactions. Then, for the resulting pseudo-plant, consisting of the original model of the multivariable process and the decoupler, SISO techniques can directly be used in designing the controllers.

The quadruple tank process, considered as a case study in this paper, is a multivariable process with a multivariable zero located in the right half plane. Apart from the non-minimum phase character, it also exhibits elegantly complex dynamics, including interactions and transmission zero [4]. This particular processes has been the focus of numerous papers, since it exhibits characteristics of interest in both control and research education. Bearing in mind that in process control industry, more than $95 \%$ of the control loops are of PI/PID (Proportional plus Integral /Proportional plus Integral plus Derivative) type, the most widely used control method for this type of processes has been the classical PID, either 
in a decentralised or in a decoupling approach [5]. For example, a recent paper compares the two approaches, while the tuning of the PI controllers is performed in several ways, ranging from direct synthesis, sequential relay with $\mathrm{ZN}$ settings, to more advanced methods such as the IMC (Internal Model Control) [5]. To reduce the interaction effects, a partial decoupling method for MIMO systems has also been proposed and implemented for the non-minimum phase quadruple tank system $[6,7]$.

Other more advanced methods have also been employed for the quadruple tank process, such as fuzzy control algorithms implemented in a decentralised version [4], with the results clearly showing the closed loop performance improvement compared to the traditional decentralised PI control. Fuzzy logic has also been used to tune a combined state-feedback sliding-mode controller for quadruple tank system [8]. The simulation results showed that the proposed version achieved better closed loop performance than the stand alone versions of state-feedback controller or sliding-mode controller. Sliding mode control has been designed and tested on an experimental setup, providing increased robustness and excellent set point tracking [9].

For the particular setup considered in this paper, the quadruple tank system from Quanser, a decentralised, decoupling and multivariable IMC strategies have been proposed [10], however the experimental results obtained showed the necessity of more complex control algorithms when stringent performance is envisaged and coupling, as well as RHP zeros need to be tackled efficiently. For this particular process, both decentralized and decoupling controls achieved poor performance for disturbance rejection tests, which motivated the application of the more advanced IMC control and even a possible future work regarding model predictive control.

The purpose of this paper is to design a simple control algorithm that is based on combining fractional order controllers with a decentralised as well as decoupling approach that allow for a SISO interpretation of the controller tuning, but that can also achieve improved performance compared to the multivariable IMC control (MIMO IMC). The fractional order PID (FOPID) controller was proposed as a generalization of the traditional integer order PID controller. The use of fractional order controllers is expected to enhance the performance of the closed loop system and increase the robustness of the system [11,12,13], being used in a wide area of applications [14].

Several fractional order techniques have been proposed in literature for controlling multivariable processes, such as the extension of the CRONE algorithm [15], MIMO-QFT robust synthesis methodology combined with CRONE control [16], sliding mode control based on the selection of a special fractional-order sliding variable [17]. Different methods for tuning multivariable fractional PID have been proposed, such as an approach to consider the tuning formulated as an $\mathrm{H} \infty$ problem with a controller structure constraint [18], the LMI (Linear Matrix Inequality) approach [19], as well as a genetic algorithm for determining the gains and orders of the fractional order PID controllers [20]. Contrary to these multivariable fractional order control algorithms, the present paper proposes simpler approaches, also based on robust fractional order control algorithms that enable the use of SISO control techniques for multivariable processes.

\section{ALTERNATIVE DESIGNS OF A FRACTIONAL ORDER CONTROLLER FOR MULTIVARIABLE PROCESSES}

The two alternative designs for the fractional order controller proposed in this paper consist ina decentralised, as well as a steady state decoupling approach.

Decentralised approach

The decentralised approach in controlling MIMO systems consists in a proper selection of the inputoutput pairings, with the purpose of dividing the initial control problem into several SISO control 
loops, while aiming to reduce the amount of interaction. The first step in the decentralised approach consists in a RGA (Relative Gain Array) analysis of the multivariable process that allows for a proper pairing of the input-output signals $[21,22]$. The next step consists in the design of the individual fractional order PI controllers for each input-output pairing by neglecting the effect of the interaction loop. The transfer function of the fractional order PI controller, proposed in this paper, is given as:

$$
H_{F O-P I}(s)=k_{p}\left(1+\frac{k_{i}}{s^{\mu}}\right)
$$

with $\mu \in(0 \div 2)$ the fractional order. To tune the fractional order PI controller, three performance specifications are imposed: a) a certain gain crossover frequency - $\left.\omega_{g c}, b\right)$ a phase margin $-\varphi_{m}$ - of the open loop system, denoted $H_{d}(s)$ and $\left.c\right)$ a robustness condition to gain variations. Considering that the open loop transfer function is written as:

$$
H_{d}(s)=H_{F O-P I}(s) H_{P}(s)
$$

where $\mathrm{H}_{\mathrm{p}}(\mathrm{s})$ is the process transfer function, the tuning of the controller is done based on the following set of equations $[23,24,25]$ :

$$
\begin{aligned}
& \left|\frac{1}{K+j L}\right|_{\omega_{g c}} \mid k_{p}\left[1+k_{i} \omega_{g c}^{-\mu}\left(\cos \frac{\pi \mu}{2}-j \sin \frac{\pi \mu}{2}\right)\right]=1 \\
& \frac{k_{i} \sin \left(\frac{\pi \cdot \mu}{2}\right)}{\omega_{g c}^{\mu}+k_{i} \cos \left(\frac{\pi \cdot \mu}{2}\right)}=\operatorname{tg}\left(\pi-\varphi_{m}-\operatorname{atan}\left(\frac{L}{K}\right)\right) \\
& \frac{\mu k_{i} \omega_{g c}^{-\mu-1} \sin \frac{\pi \mu}{2}}{1+2 k_{i} \omega_{g c}^{-\mu} \cos \frac{\pi \mu}{2}+k_{i}^{2} \omega_{g c}^{-2 \mu}}-\frac{\dot{L} K-L \dot{K}}{L^{2}+K^{2}}=0
\end{aligned}
$$

where $\mathrm{K}$ is the real part and $\mathrm{L}$ is its imaginary part of the process $\mathrm{HP}_{\mathrm{P}}\left(j \omega_{g c}\right)$. To simplify the computation of the fractional order PI controller parameters, the values for $\mathrm{k}_{\mathrm{i}}$ and $\mu$ are determined graphically using (4) and (5) $[23,24,25]$, while $\mathrm{k}_{\mathrm{p}}$ is then computed using (3).

\section{Decoupling approach}

In case of a highly coupled MIMO system, the decentralised approach may result in poor closed loop performance due to the multiple input-output interactions. A decoupling solution could then be used instead. In this paper, a steady state decoupling is employed. Given the $n \times n$ MIMO system:

$$
G_{p}(s)=\left(\begin{array}{cccc}
H_{p 11}(s) & H_{p 12}(s) & \ldots & H_{p 1 n}(s) \\
H_{p 21}(s) & H_{p 22}(s) & \ldots & H_{p 2 n}(s) \\
\ldots & \ldots & \ldots & \ldots \\
H_{p n 1}(s) & H_{p n 2}(s) & \ldots & H_{p n n}(s)
\end{array}\right)
$$


the steady state decoupler is the inverse of the process transfer function gain matrix in (6), denoted as $G_{m}^{\#}$. The steady state decoupled process is then computed as:

$$
G_{D}(s)=G_{m}^{\#}\left(\begin{array}{cccc}
H_{p 11}(s) & H_{p 12}(s) & \ldots & H_{p 1 n}(s) \\
H_{p 21}(s) & H_{p 22}(s) & \ldots & H_{p 2 n}(s) \\
\ldots & \ldots & \ldots & \ldots \\
H_{p n 1}(s) & H_{p n 2}(s) & \ldots & H_{p n n}(s)
\end{array}\right)
$$

The tuning of the fractional order PI controllers is then performed for each diagonal element in the decoupled process $\mathrm{G}_{D}(\mathrm{~s})$ using the same tuning procedure based on (3)-(5). The final multivariable FO-PI controller computed as:

$$
G_{C}(s)=G_{m}^{\#}\left(\begin{array}{cccc}
H_{F O-P I_{1}}(s) & 0 & \ldots & 0 \\
0 & H_{F O-P I_{2}}(s) & \ldots & 0 \\
\ldots & \ldots & \ldots & 0 \\
0 & 0 & \ldots & H_{F O-P I_{n}}(s)
\end{array}\right)
$$

\section{CASE STUDY. CONTROL STRATEGIES FOR NON_MINIMUM PHASE QUADRUPLE TANK SYSTEM}

The schematic representation of the quadruple water tanks system is given in Fig. 1. The system is a multivariable one, with two inputs, the voltages applied to the two pumps, denoted as $V_{p l}(t)$ and $\mathrm{V}_{\mathrm{p} 2}(\mathrm{t})$, and two outputs, the water levels of the lower tanks, Tank2 and Tank4, denoted as $\mathrm{L}_{2}(\mathrm{t})$ and $\mathrm{L}_{4}(\mathrm{t})$, respectively. There is a strong coupling effect between the inputs and the outputs. Such a coupling may be observed in Tank2 which has two inputs: the flow from Pump1 $\left(\mathrm{V}_{\mathrm{p} 1}(\mathrm{t})\right)$ through Out2, marked with dashed red line, and the flow from Pump2 $\left(\mathrm{V}_{\mathrm{p} 2}(\mathrm{t})\right)$ through Out1, denoted with green continuous line, that is the output flow from Tank1). Hence, the controlled level in Tank $2\left(\mathrm{~L}_{2}\right)$ is influenced by the two inputs.

By a simple adjustment of the percentage of water flow from each input, one can change the system for having minimum phase or non-minimum phase dynamics [10]. The configuration used in this paper and indicated in Figure 1 is a non-minimum phase one, with a greater flow coming from Pump2, via Tank1, into Tank2, in comparison with the flow coming directly from Pump1. This is due to the fact that the outlet diameter Out1 is bigger than the diameter Out2, while the outgoing orifices from each tank Do1, Do2, Do3 and Do4 have the same diameter. A similar situation occurs in the case of Tank4. Then, the dominant flow in Tank2 and Tank4 comes from the manner in which the physical coupling is implemented via the choice of the setup $[26,27]$.

The model transfer function matrix has been previously determined experimentally to be [10]:

$$
G(s)=\left[\begin{array}{cc}
\frac{1.64}{18.43 s+1} & \frac{2.49}{178.8 s^{2}+26.74 s+1} \\
\frac{2.56}{172.2 s^{2}+27.6 s+1} & \frac{1.28}{15.92 s+1}
\end{array}\right]
$$

The transmission zeros for the quadruple water tanks system are: $\mathrm{z}_{1}=-0.26 ; \mathrm{z}_{2}=0.07 ; \mathrm{z}_{3}=-0.06 ; \mathrm{z}_{4}=-$ 0.05 . Due to the positive zero $z_{2}=0.07$, the system is non-minimum phase. 


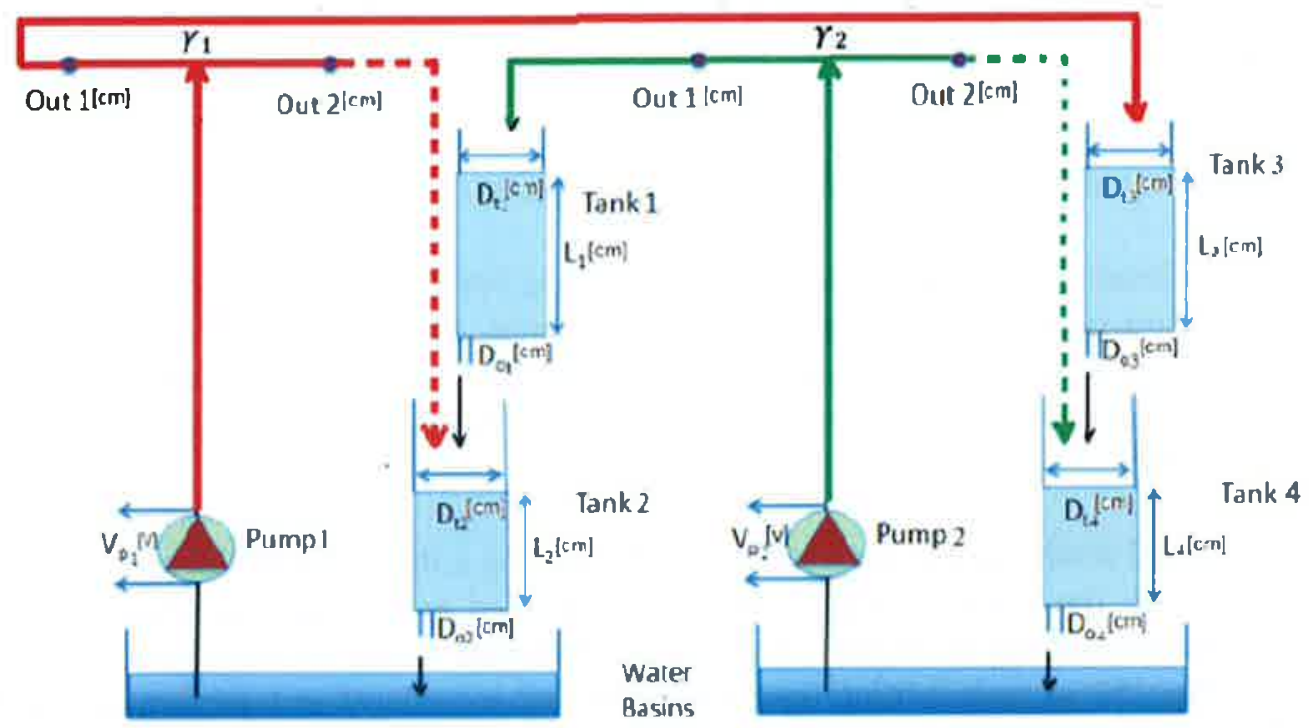

Fig. 1. Schematic diagram of the quadruple water tanks system

A simple RGA analysis show that for the configuration previously described the following RGA values are obtained [10]:

$$
\Lambda=\left[\begin{array}{cc}
-0.49 & 1.49 \\
1.49 & -0.49
\end{array}\right]
$$

According to (10), t1-2/2-1 pairing is selected and two FO-PI controllers are then computed. The following performance specifications are imposed for the two loops: $\omega_{g c 1}=0.03, \varphi_{m 1}=70^{\circ}$ and $\omega_{g c 2}=0.03, \varphi_{m 2}=70^{\circ}$. The resulting fractional order PI controllers, to be used in the decentralised approach are:

$$
\left\{\begin{array}{l}
H_{F O-P I_{1}}(s)=0.3\left(1+\frac{0.024}{s^{1.16}}\right) \\
H_{F O-P I_{2}}(s)=0.29\left(1+\frac{0.025}{s^{1.16}}\right)
\end{array}\right.
$$

To tune the fractional order controllers for the decoupling control strategy, the decoupler was first computed as:

$$
G_{m}^{\#}=\left(\begin{array}{cc}
-0.3 & 0.58 \\
0.6 & -0.38
\end{array}\right)
$$

Similar performance specifications were imposed to design the fractional order controllers for the decoupling strategy, $\omega_{g c l}=0.02, \varphi_{m 1}=70^{\circ}$ and $\omega_{g c 2}=0.02, \varphi_{m 2}=70^{\circ}$, in order to obtain similar closed loop performance in terms of overshoot and settling time. The two fractional order controllers are:

$$
H_{F O-P I_{1}}(s)=0.53\left(1+\frac{0.022}{s^{1.16}}\right)
$$




$$
H_{F O-P I_{2}}(s)=0.6\left(1+\frac{0.018}{s^{1.17}}\right)
$$

with the final multivariable FO-PI controller determined using (8).

To compare the results, a multivariable IMC strategy has been designed according to [10], to yield similar closed loop performance in terms of settling time, as compared to the decentralised and decoupling fractional order control algorithms given by (11) and (13)-(14), respectively. The closed loop simulation results, considering step changes in the reference signals for the levels $\mathrm{L}_{2}$ and $\mathrm{L}_{4}$, are given in Fig. 2 and 3.

Since the simplified model in (9) was obtained by linearizing a nonlinear model around the operating point of $10 \mathrm{~cm}$ [10], the results in Fig. 2 and 3 are regarded as nominal operating conditions. The decentralised and decoupling fractional order control strategies ensure no overshoot and 150 seconds settling time. The 150 seconds settling time will be further considered as a performance criteria. The MIMO IMC algorithm ensures the same settling time, but with an overshoot of $25 \%$. It must be noted here that zero overshoot for the MIMO IMC strategy is possible to be obtained at the expense of a major increase in the settling time. In terms of interaction, the MIMO IMC offers the best results, however this is valid under the assumption of a perfect model. Among the fractional order control strategies, the decoupling approach provides better interaction responses than the decentralised control algorithm.

To test the robustness of the designed controller, similar step changes in the reference signals were considered, but with a variation of $30 \%$ of the gains and time constants of the process in (9):

$$
G(s)=\left[\begin{array}{cc}
\frac{2.14}{23.96 s+1} & \frac{3.24}{232 s^{2}+26.74 s+1} \\
\frac{3.33}{230 s^{2}+27.6 s+1} & \frac{1.66}{21 s+1}
\end{array}\right]
$$

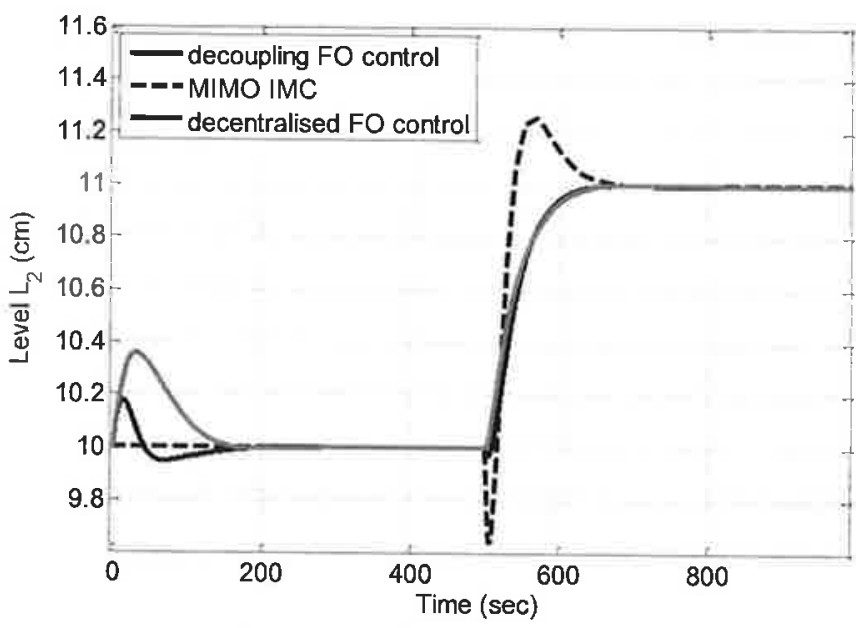

Fig. 2. Comparative nominal closed loop simulation results considering a step change in the reference signal for $L_{2}$ 


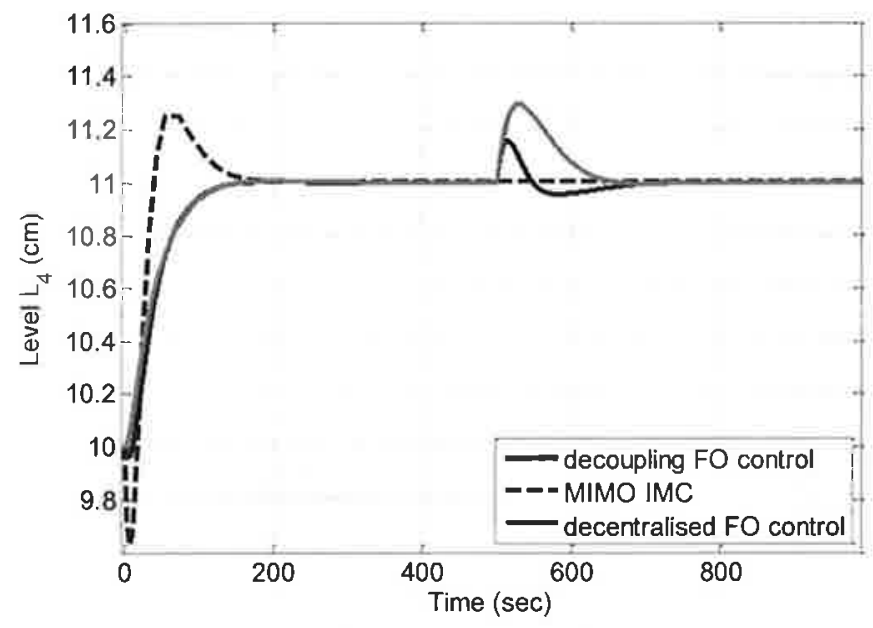

Fig. 3. Comparative nominal closed loop simulation results considering a step change in the reference signal for $\mathrm{L}_{4}$

The closed loop comparative robustness simulation results are indicated in Fig. 4 and 5. As noted from the two figures, for the fractional order control strategies, the $30 \%$ change in the modeling parameters do not affect significantly the closed loop performance results, with no overshoot and a smaller settling time below 120 seconds for both outputs. Considering the performance criteria of 150 seconds maximum settling time, both the decentralised and the decoupling FO controllers meet this requirement. The robustness of the decentralized and decoupling control strategies are almost identical in terms of reference tracking. On the other hand, the MIMO IMC results show a degradation of the closed loop performance, with a slight increase in the settling time of 170 seconds, but a significant increase of the overshoot accounting to $50 \%$. The maximum amplitudes of the interaction responses show that the MIMO IMC and the decoupling FO controllers have similar performance, with the decentralised FO controllers behaving the poorest. The settling time is however $50 \%$ larger with the MIMO IMC (150 seconds) compared to the decentralised and decoupling FO controllers. Overall, the proposed fractional order decentralised and decoupling strategies offer an increased robustness as compared to the previously proposed MIMO IMC algorithm.

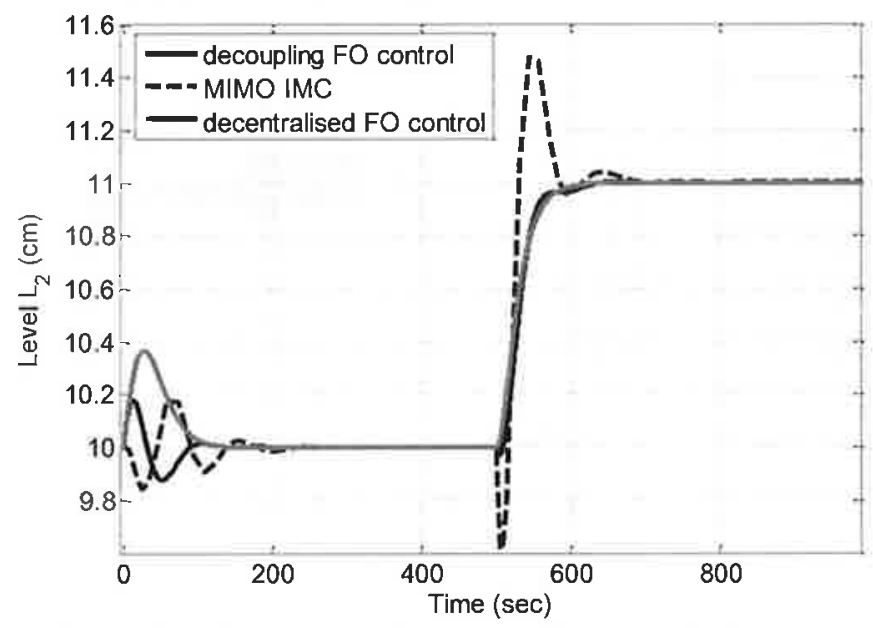

Fig. 4. Comparative robust closed loop simulation results considering a step change in the reference signal for $\mathrm{L}_{2}$ 


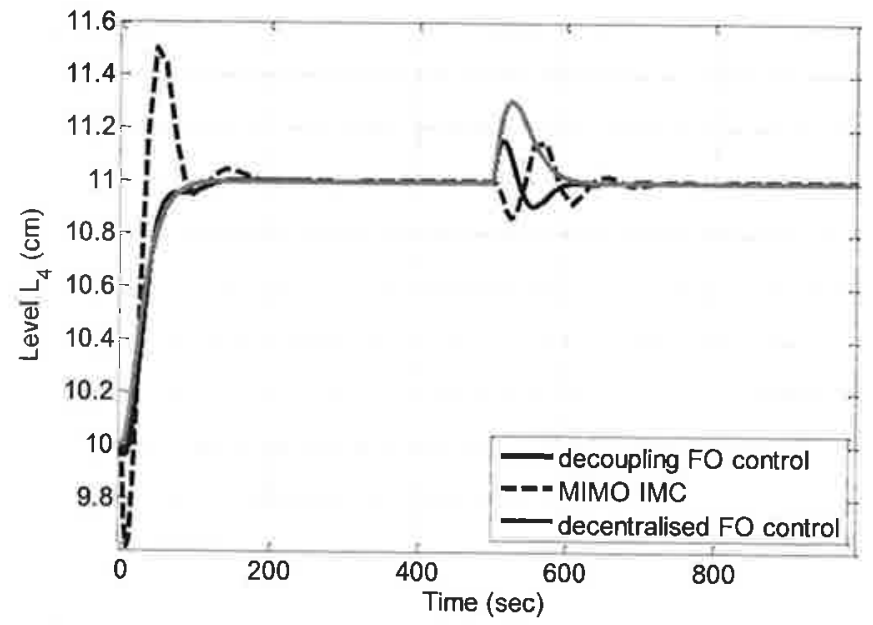

Fig. 5. Comparative robust closed loop simulation results considering a step change in the reference signal for $\mathrm{L}_{4}$

Previous results [10] showed that poor disturbance rejection performance was achieved when using classical integer order PID controllers in a decentralised or decoupling approach, which justified the application of the more advanced MIMO IMC control. Fig. 6 and 7 present the disturbance rejection tests, considering the nominal conditions, while Fig. 8 and 9 present the same disturbance rejection tests in the case of the modeling errors in (15). The simulation results show that the MIMO IMC and the decoupling fractional order controller are outperformed in terms of settling times by the decentralised fractional order controller. Also, the MIMO IMC is more oscillating with increased amplitudes compared to the decoupling fractional order controller.

To evaluate the disturbance rejection tests, the following performance index was used:

$J=\sum_{t=0}^{\infty}\left(r_{i}(t)-y_{i}(t)\right)^{2}$, with $\mathrm{i}=1,2$

where $r_{\mathrm{i}}$ is the setpoint for the corresponding output signal $y_{i}$ (either tank levels $\mathrm{L}_{2}$ or $\mathrm{L}_{4}$ ).

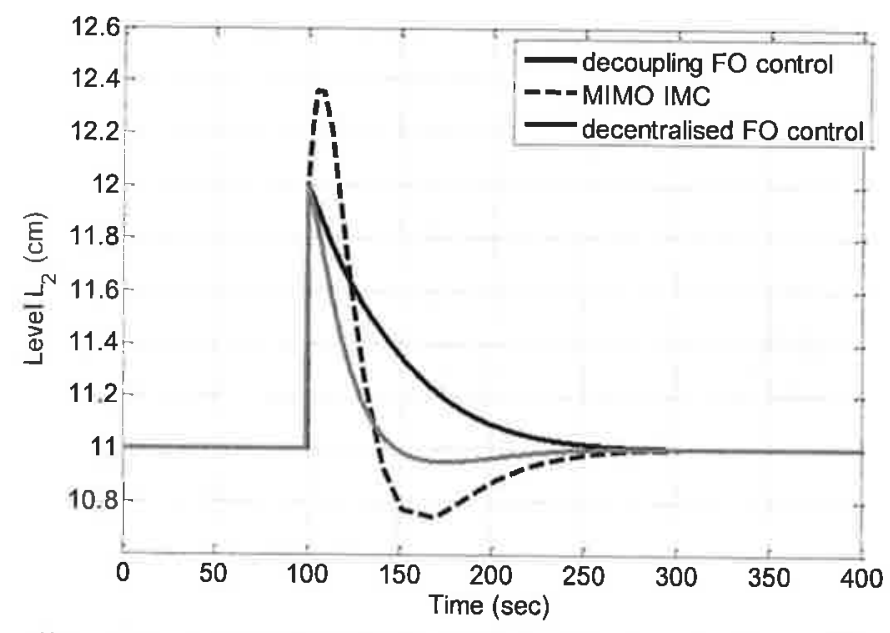

Fig. 6. Comparative disturbance rejection tests considering nominal conditions and a step change in the reference signal for $\mathrm{L}_{2}$ 


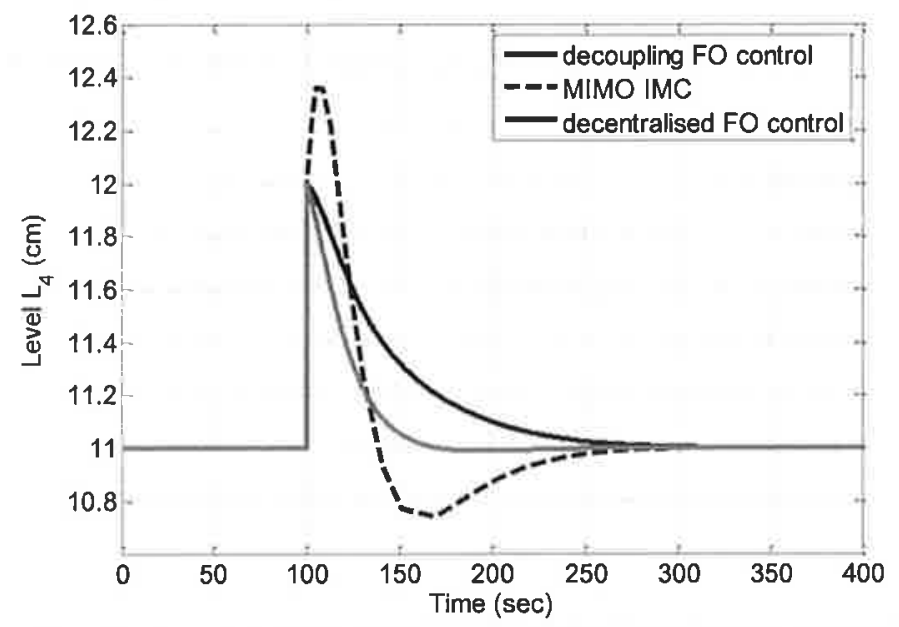

Fig. 7. Comparative disturbance rejection tests considering nominal conditions and a step change in the reference signal for $\mathrm{L}_{4}$

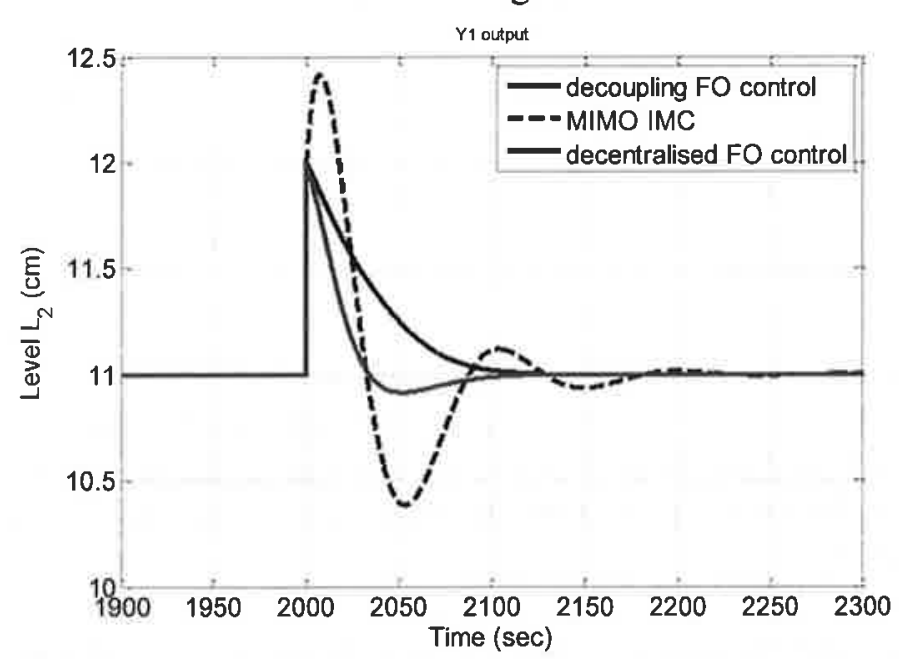

Fig. 8. Comparative disturbance rejection tests considering modelling errors and a step change in the reference signal for $\mathrm{L}_{2}$

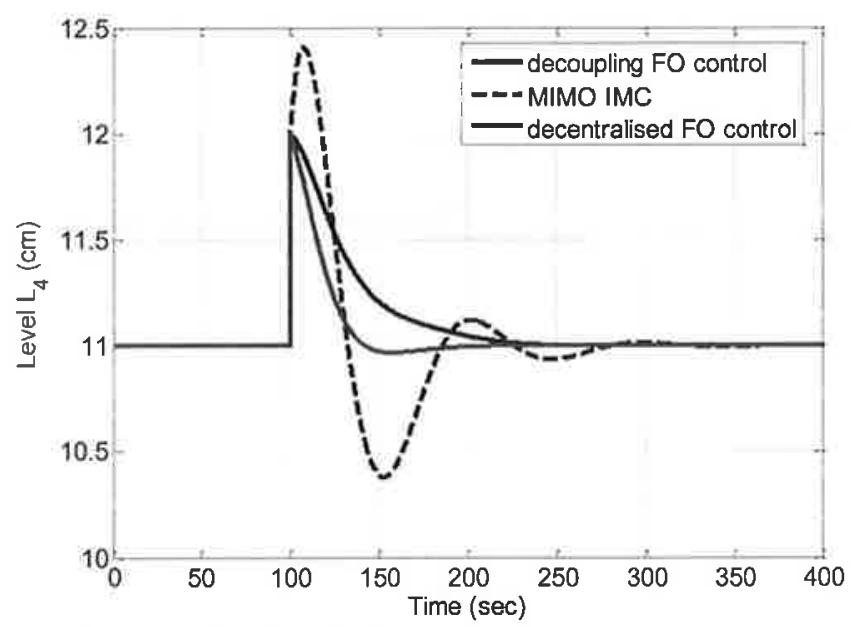

Fig. 9. Comparative disturbance rejection tests considering modelling errors and a step change in the reference signal for $\mathrm{L}_{4}$ 
The computed values are given in Table 1 and show that the proposed fractional order control strategies outperform the MIMO IMC in terms of disturbance rejection, both under nominal as well as modelling errors.

\begin{tabular}{|l|l|l|l|l|}
\hline Control strategy & \multicolumn{2}{|l|}{ Output y1 } & Output y2 \\
\cline { 2 - 5 } & Nominal & Modelling errors & Nominal & Modelling errors \\
\hline $\begin{array}{l}\text { Decentralised } \\
\text { fractional order } \\
\text { control }\end{array}$ & $\mathrm{J}=10.35$ & $\mathrm{~J}=9.37$ & $\mathrm{~J}=11.35$ & $\mathrm{~J}=10.03$ \\
\hline $\begin{array}{l}\text { Decoupling } \\
\text { fractional order } \\
\text { control }\end{array}$ & $\mathrm{J}=23.6$ & $\mathrm{~J}=19.57$ & $\mathrm{~J}=23.6$ & $\mathrm{~J}=19.3$ \\
\hline MIMO IMC & $\mathrm{J}=34.23$ & $\mathrm{~J}=43.72$ & $\mathrm{~J}=34.22$ & $\mathrm{~J}=43.31$ \\
\hline
\end{tabular}

Table 1. Performance index for the disturbance rejection tests

\section{EXPERIMENTAL RESULTS}

The two fractional order control strategies described above have been tested on the coupled tanks system by Quanser. Figures 10 and 11 show the comparative closed loop tests for both the decentralised and the decoupling fractional order control strategies. The case study considered here consists in a step change for the $\mathrm{L}_{2}$ reference from $9 \mathrm{~cm}$ to $11 \mathrm{~cm}$, thus near the linearization point. The $\mathrm{L}_{4}$ reference signal is kept at $10 \mathrm{~cm}$.

In terms of interaction, the decoupling fractional order control strategy performs better than the decentralised fractional order control algorithm. Using the same performance index as in (16) for the interaction responses yields a value of $\mathrm{J}=9.14$ for the decoupled version, while for the decentralised control strategy, $\mathrm{J}=21.26$. This suggests that the decoupled control algorithm ensures a $50 \%$ reduction of the interaction responses.

In terms of reference tracking, the experimental results in figure 10 show that the two fractional order control strategies achieve similar performance. The settling time in both cases is 150 seconds, as obtained in the simulation results presented in the previous section, with a slight increase in the overshoot of $2 \%$.

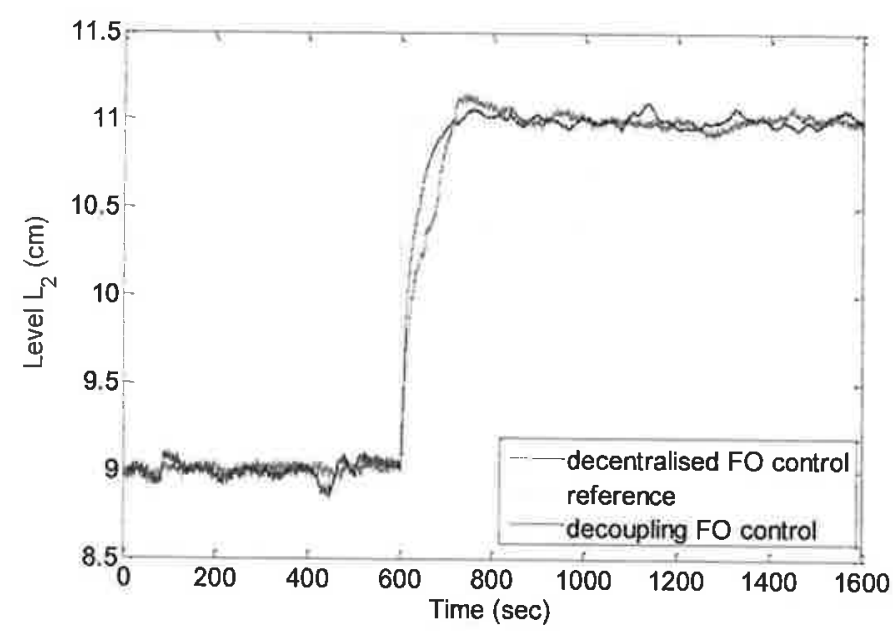

Fig. 10. Comparative closed loop experimental results for reference tracking 


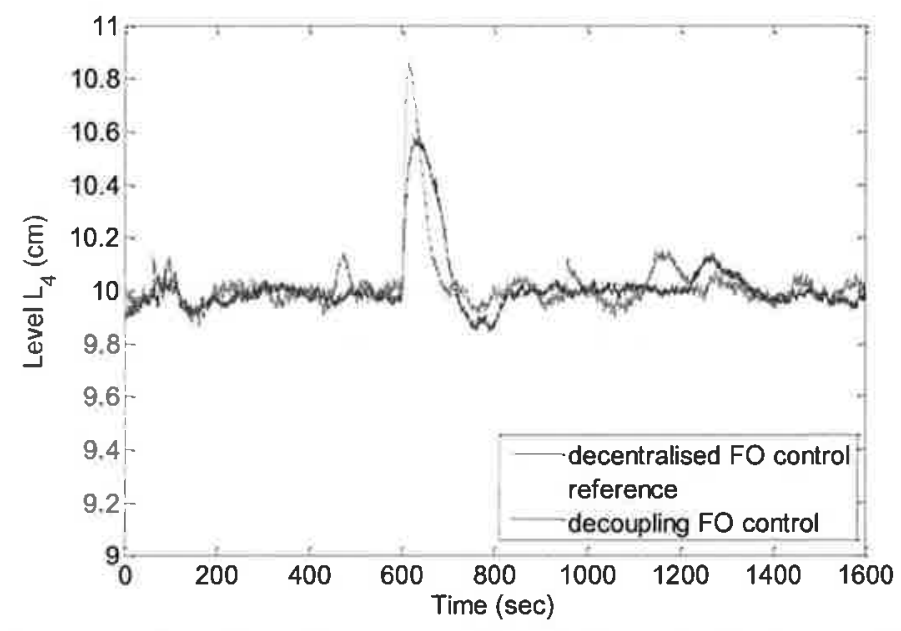

Fig. 11. Comparative closed loop experimental results for interaction response

\section{CONCLUSIONS}

This paper presented two alternative solutions for controlling non-minimum phase systems and significant coupling. The previous traditional decentralised and decoupling strategies applied for the presented case study, the quadruple tank system, have shown the necessity for an advanced control solution, such as the MIMO IMC. The alternative solutions proposed in this paper consist in decentralised and decoupling fractional order control strategies. The simulation results prove that the proposed multivariable fractional order control algorithms outperform the MIMO IMC solution previously proposed, in terms of closed loop performance, disturbance rejection, both under nominal conditions, as well as modelling errors.

The experimental results considering the decoupled and decentralised fractional order control strategies are in good agreement with the closed loop simulation results. The settling time and the overshoot obtained on the experimental quadruple tank system meet the performance criteria specified for the simulated closed loop system. The decoupled fractional order control algorithm achieves similar performance in terms of reference tracking when compared to the decentralised fractional order control strategy. The most important contribution of the decoupled fractional order control algorithm is the $50 \%$ reduction in the interaction response, compared to the decentralised approach.

\section{Acknowledgement}

This work was supported by a grant of the Romanian National Authority for Scientific Research, CNCS - UEFISCDI, project number TE 59/2013.

\section{References}

1. Bequette W (2003) Process Control: Modeling, Design and Simulation, Prentice Hall Professional.

2. Kantera JM, Soroushb M and Seidera WD (2002) Nonlinear feedback control of multivariable nonminimum-phase processes. Journal of Process Control 12:667-686

3. Astrom KJ, Johansson AH and Wang QG (2002) Design of decoupled PI controllers for two by two system. IEE Proceedings on Control Theory and Applications 149: 74-81

4. Suja Mani Malar R and Thyagarajan T (2008) Design of decentralized fuzzy controllers for quadruple tank process. International Journal of Computer Science and Network Security 8: 163-168

5. Ramadevi C and Vijayan V (2014) Design of decoupled pi controller for quadruple tank system. International Journal of Science and Research 3: 318-323 
6. Garelli F, Mantz R J and Battista HD (2006) Partial decoupling of non-minimum phase processes with bounds on the remaining coupling. Chemical Engineering Science 61: 7706-7716

7. Garelli F, Mantz RJ and Battista HD (2006) Limiting interactions in decentralized control of MIMO system. Journal of Process Control 16: 473-483

8. Mirakhorli E and Farrokhi M (2011) Sliding-mode state-feedback control of non-minimum phase quadruple tank system using fuzzy logic. Proceedings of the 18th IFAC World Congress 1: 1354613551

9. Pani Biswas P et al. (2009) Sliding mode control of quadruple tank process. Mechatronics 19: 548561

10. Maxim A et al. (2013) Multivariable model-based control strategies for level control in a quadruple tank process. Proceedings of the 17th International Conference on System Theory, Control and Computing Joint Conference 1: 343 - 348

11. Podlubny I (1999) Fractional-order systems and PI $\lambda D \mu$ - controllers. IEEE Transactions on Automatic Control 44: 208-214

12. Oustaloup A (1991) La Commande CRONE: Commande Robuste d'Ordre Non Entier. Paris: Hermes

13. Li HS, Luo Y and Chen YQ (2010) A fractional order proportional and derivative (FOPD) motion controller: tuning rule and experiments. IEEE Transactions on Control Systems Technology 18: 516520

14. Gutiérrez RE, Rosário JM and Machado JT (2010) Fractional order calculus: basic concepts and engineering applications. Mathematical Problems in Engineering article ID: 375858

15. Gruel DN, Lanusse P and Oustaloup A (2009) Robust control design for multivariable plants with time-delays. Chemical Engineering Journal 146: 414-427

16. Yousfi $\mathrm{N}$ et al. (2012) Comparison between $\mathrm{H} \infty$ and CRONE control combined with QFT approach to control multivariable systems in path tracking design, International Journal of Computer Applications 45:1-9

17. Pisano A et al. (2010) Sliding mode control approaches to the robust regulation of linear multivariable fractional-order dynamics. International Journal of Robust and Nonlinear Control 20: 2045-2056

18. Chenikher S, Abdelmalek S and Sedraoui M (2012) Control of uncertainly multi-variable system with fractional PID, Proceedings of the 16th IEEE Mediterranean Electrotechnical Conference 1: $1079-1082$

19. Song X et al. (2011) Multivariable fractional order PID controller design via LMI approach. Proceedings of the 18th IFAC World Congress 1: 13960-13965

20. Moradi M (2014) A genetic-multivariable fractional order PID control to multi-input multi-output processes. Journal of Process Control 24: 336-343

21. Bristol E (1966) On a new measure of interaction for multivariable process control. IEEE Transactions on Automatic Control 11: 133-134

22. Moaveni B and Khaki-Sedigh A (2007) Input-output pairing for nonlinear multivariable systems. Journal of Applied Science 7: 3492-3498

23. Muresan CI et al. (2013) Development and implementation of an FPGA based fractional order controller for a DC motor. Mechatronics 23: 798-804

24. Monje CA et al. (2010) Fractional order Systems and Controls: Fundamentals and Applications. London: Springer-Verlag

25. Muresan CI (2014) Fractional Calculus: From Simple Control Solutions to Complex Implementation Issues, chapter in Machado JAT, Baleanu D and Luo A (eds) Discontinuity and Complexity in Nonlinear Physical Systems, Nonlinear Systems and Complexity 6: 113-134, Switzerland: Springer

26. Johansson KJ (2000) The quadruple-tank process. A multivariable laboratory process with an adjustable zero. IEEE Transactions on Control Systems Technology 8: 456-465 
27. Johansson KJ et al. (1999) Teaching multivariable control using the quadruple-tank process. Proceedings of the 38th IEEE Conference on Decision and Control 1: 807-812 
